\title{
Thermally-inverted strain-optical and stress-optical behavior of poly(4-methyl-1-pentene) in rubbery state
}

\author{
S. Gupta, I. Offenbach, R. A. Weiss, M. Cakmak* \\ Department of Polymer Engineering, University of Akron, OH, United States 44325
}

Received 4 February 2019; accepted in revised form 30 April 2019

\begin{abstract}
The unique temperature-dependent strain-optical and stress-optical behavior of poly(4-methyl-1-pentene) (P4MP) is reported during uniaxial deformation in the rubbery state. For P4MP the birefringence is found to increase with an increase in deformation temperature, which is opposite to the behavior of other polyolefins such as polypropylene and polyethylene. Real-time stress-strain-birefringence measurements are supplemented with offline wide-angle X-ray diffraction (WAXD) and infrared (FTIR) measurements.
\end{abstract}

Keywords: material testing, poly(4-methyl-1-pentene) p4mp, birefringence, strain-optic, stress-optic

\section{Introduction}

Poly(4-methyl-1-pentene) (P4MP) has recently attracted renewed attention as a promising polymer for high-temperature energy storage applications $[1,2]$. Dielectric polymer films are commercially fabricated by either melt-processing or solution-processing followed by polymer chain orientation through uniaxial or biaxial stretching. In our recent work [1] we showed a 4-fold increase in the dielectric breakdown strength of P4MP upon solution-processing and biaxially stretching thin P4MP films. When those solution-processed films were uniaxially stretched at different temperatures, we observed a unique temperature-dependent strain-optical behavior which has never been reported for P4MP. This behavior is very interesting from a scientific viewpoint along with some practical implications. For example, a major commercial application of P4MP includes its use as an optically clear, low-birefringence film for packaging solutions and optical lenses. Standard meltprocessing methods such as extrusion, injectionmolding and blow-molding generate considerable polymer chain orientation which affects the optical properties of the final part such as birefringence [3, 4]. Therefore understanding the mechano-optical response of P4MP can enable manufacturing of films with improved properties as well as optimize the processing conditions. In this paper we discuss the unique mechano-optical behavior of thin P4MP films observed during uniaxial deformation in the rubbery state, and propose a deformation mechanism based on those results. Real-time stress-strain-birefringence measurements are performed using an instrumented uniaxial mechano-optical stretching platform. These measurements are augmented with offline wide-angle X-ray diffraction (WAXD) and Fourier-transform infrared spectroscopy (FTIR).

\section{Experimental details}

\subsection{Materials}

A commercial-grade P4MP (TPX ${ }^{\mathrm{TM}}$ MX002O) was provided by Mitsui Chemicals. Cyclopentane was purchased from Fisher Scientific Co. (99\% pure, AC111480025). The polymer was vacuum-dried overnight at $60^{\circ} \mathrm{C}$ before use and the solvent was used as-received. 


\subsection{Sample preparation}

Unoriented $20 \mu \mathrm{m}$ thick P4MP films were prepared by solution-casting on a roll-to-roll (R2R) platform using Cyclopentane as a solvent on a PET substrate. The method is described in detail elsewhere [1]. Dumbbell shaped specimens were punched out using a die from the cast film for further characterization.

\subsection{Characterization}

Polymer specimens were uniaxially stretched in the rubbery state at various temperatures at a stretch rate of $10 \mathrm{~mm} / \mathrm{min}$. An instrumented real-time mechanooptical measurement platform was used to investigate the deformation behavior of P4MP thin films. Details of the measurement technique are discussed elsewhere [5, 6]. In brief, the specimen was clamped to load cells in the uniaxial stretching machine and enclosed within a thermal chamber. The stress-strain curves were calculated from the measured force and the true cross-sectional area of the deformed specimen by Equations (1)-(3):

$$
\begin{aligned}
& \sigma_{\mathrm{T}}=\frac{F}{W_{\mathrm{t}} D_{\mathrm{t}}}=\frac{F}{\frac{W_{\mathrm{t}}^{2}}{W_{0}} D_{0}} \\
& \varepsilon_{\mathrm{T}}=\frac{L_{\mathrm{t}}-L_{0}}{L_{0}}=\left(\frac{W_{0}}{W_{\mathrm{t}}}\right)^{2}-1 \\
& \varepsilon_{\mathrm{H}}=\ln \left(\frac{L_{\mathrm{t}}}{L_{0}}\right)=\ln \left(\frac{W_{0}}{W_{\mathrm{t}}}\right)^{2}=\ln \left(1+\varepsilon_{\mathrm{T}}\right)
\end{aligned}
$$

where $F, \sigma_{\mathrm{T}}, \varepsilon_{\mathrm{T}}$, and $\varepsilon_{\mathrm{H}}$ are the force, true stress, true strain and Hencky strain, respectively. $D, W$ and $L$ are the thickness, width and length, respectively. The subscript ' 0 ' indicates initial values whereas subscript ' $t$ ' indicates real-time values. Equations (1)-(3) were determined by measuring the real-time width $\left(W_{\mathrm{t}}\right)$ of the specimen and assuming incompressibility $\left(L_{\mathrm{t}} / L_{0}=\left(W_{0} D_{0}\right) /\left(W_{\mathrm{t}} D_{\mathrm{t}}\right)\right)$ and transverse isotropy $\left(W_{\mathrm{t}} / W_{0}=D_{\mathrm{t}} / D_{0}\right)$. These assumptions have been checked in an earlier publication on polypropylene (PP) and error committed was found to be within 4\% of actual [6]. However, these assumptions were violated for P4MP thin films [7]. The Poisson ratios $\left(v_{13}\right.$ and $v_{12}$, where $1,2,3$ refer to the directions of stretch/length, width and thickness, respectively) were calculated to be not only less than 0.5 (implying a compressible system) but also different from each other. The stress-strain curves for P4MP thin films were calculated by assuming that the ratio of
Poisson ratios $\left(v_{13} / v_{12}\right)$ is constant under isothermal deformation, which allowed us to back-calculate the real-time thickness $\left(D_{\mathrm{t}}\right)$. Details are provided elsewhere [7]. A visible wavelength light source was used to measure the optical retardation $(\Gamma)$ through the polymer film. The in-plane birefringence $\left(\Delta n_{12}\right)$ was calculated from Equation (4):

$\Delta n_{12}=n_{1}-n_{2}=\frac{\Gamma}{D_{\mathrm{t}}}$

where $n_{1}$ and $n_{2}$ are the refractive indices in the stretch direction and transverse (width) direction, respectively.

Thermal characterization was performed using a TA Instruments Q-200 differential scanning calorimetry (DSC). DSC was used to measure the glass transition temperature $\left(T_{\mathrm{g}}\right)$, the melting point $\left(T_{\mathrm{m}}\right)$ and the heat of fusion $\left(\Delta H_{\mathrm{f}}\right)$ of the polymer films. Before the measurements, temperature calibration was performed using an Indium standard $\left(T_{\mathrm{m}}=156.6^{\circ} \mathrm{C}\right)$. $T_{\mathrm{g}}$ was defined as the inflection point in the change of the heat capacity associated with the glass transition. The melting point $\left(T_{\mathrm{m}}\right)$ was defined as the peak of the melting endotherm. Mass fraction crystallinity of the polymer $\left(\phi_{\mathrm{m}}\right)$ was calculated from the ratio of $\Delta H_{\mathrm{f}} / \Delta H^{0}$, where $\Delta H_{\mathrm{f}}$ was the area under the DSC melting endotherm and $\Delta H^{0}$ is the enthalpy of fusion for a $100 \%$ crystalline polymer, which is reported to be $61.85 \mathrm{~J} / \mathrm{g}$ for P4MP with a tetragonal crystal structure [8].

The polymer crystal structure was characterized with wide-angle X-ray diffraction (WAXD) using a Bruker AXS D8 Goniometer with a $\mathrm{CuK}_{\alpha}$ radiation $(\lambda=$ $0.1542 \mathrm{~nm}$ ). WAXD diffractograms were used to measure any changes in the crystal structure upon stretching, and also to calculate the Hermans orientation function of polymer chains in the crystalline regions $\left(f_{\mathrm{c}}\right)$ using the Equation (5):

$f_{\mathrm{c}, \mathrm{z}}=\frac{1}{2}\left[3\left\langle\cos ^{2} \chi_{\mathrm{c}, \mathrm{z}}\right\rangle-1\right]$

where $\left\langle\cos ^{2} \chi_{\mathrm{c}, \mathrm{z}}\right\rangle$ represents the mean-square cosine (average over all crystallites) of the angle $\chi$ between a given crystal axis and the reference $z$-axis (machine stretch direction in the present case). For P4MP $\left\langle\cos ^{2} \chi_{c, z}\right\rangle$ was calculated using the Equation (6) [9]:

$\left\langle\cos ^{2} \chi_{c, z}\right\rangle=1-2\left\langle\cos ^{2} \chi_{200, z}\right\rangle$ 
$\left\langle\cos ^{2} \chi_{\mathrm{hkl}, \mathrm{z}}\right\rangle$ was calculated by measuring the intensity diffracted by the $(h k l)$ planes and using the following Equation (7):

$$
\left\langle\cos ^{2} \chi_{\mathrm{hkl}, \mathrm{z}}\right\rangle=\frac{\int_{0}^{\pi / 2} I(\chi) \sin \chi \cos ^{2} \chi}{\int_{0}^{\pi / 2} I(\chi) \sin \chi}
$$

The stretched polymer films were also characterized by Fourier transform infrared spectroscopy (FTIR) using a Thermo Scientific Nicolet 380 spectrometer. The absorption spectra were measured from 525 $4500 \mathrm{~cm}^{-1}$ using 64 scans with a resolution of $4 \mathrm{~cm}^{-1}$.

\section{Results and discussion}

The physical and thermal properties of P4MP are summarized in Table 1, and the DSC endotherm is shown in Figure 1. Uniaxial stretching of P4MP film was conducted at series of temperatures below the melting point $22,50,100$ and $150{ }^{\circ} \mathrm{C}$ with a constant stretch rate of $10 \mathrm{~mm} / \mathrm{min}$. These temperatures are indicated by vertical dotted lines in Figure 1,

Table 1. Physical and thermal properties of P4MP.

\begin{tabular}{|l|c|l|c|c|c|c|}
\hline Polymer & $\begin{array}{c}\boldsymbol{M}_{\mathbf{v}}{ }^{\mathbf{a}} \\
{[\mathbf{k D a}]}\end{array}$ & $\begin{array}{c}\boldsymbol{\rho}^{\mathbf{b}} \\
{\left[\mathbf{g} / \mathbf{c m}^{\mathbf{3}}\right]}\end{array}$ & $\begin{array}{c}\boldsymbol{T}_{\mathbf{g}}^{\mathbf{c}} \\
{\left[{ }^{\circ} \mathbf{C}\right]}\end{array}$ & $\begin{array}{c}\boldsymbol{T}_{\mathbf{m}, \text { onset }} \\
{\left[{ }^{\circ} \mathbf{C}\right]}\end{array}$ & $\begin{array}{c}\boldsymbol{T}_{\mathbf{m}}{ }^{\mathbf{c}} \\
{\left[{ }^{\circ} \mathbf{C}\right]}\end{array}$ & $\begin{array}{c}\boldsymbol{\phi}_{\mathbf{m}} \\
{[\mathbf{\%}]}\end{array}$ \\
\hline P4MP & 115 & 0.834 & 26 & 160 & 226 & 18 \\
\hline
\end{tabular}

${ }^{\mathrm{a}} M_{\mathrm{v}}$ : viscosity-average molecular weight; calculated from $[\eta]=$ $K \cdot M_{\mathrm{v}}{ }^{\alpha}$ where $K=1.94 \cdot 10^{-5}$ and $\alpha=0.81[10]$. [ $\left.\eta\right]$ was measured in decalin at $135^{\circ} \mathrm{C}$.

${ }^{\mathrm{b}} \rho=$ density; from as-received polymer pellets.

${ }^{\mathrm{c}} T_{\mathrm{g}}=$ glass-transition; $T_{\mathrm{m}, \text { onset }}=$ onset of melting; $T_{\mathrm{m}}=$ melting peak; $\phi_{\mathrm{m}}=$ crystallinity; from DSC on unstretched films, heat cycle at $10^{\circ} \mathrm{C} / \mathrm{min}$.

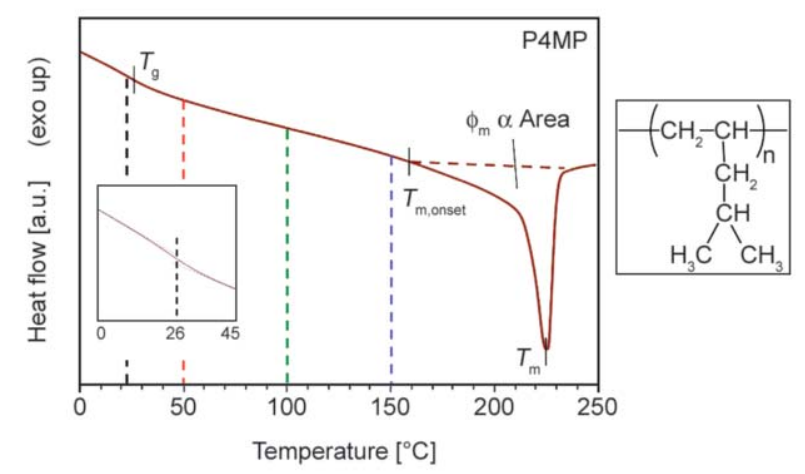

Figure 1. DSC endotherm for P4MP obtained from solutioncast unstretched film. The symbols indicate the following: $T_{\mathrm{g}}-$ glass transition, $T_{\mathrm{m} \text {,onset }}-$ onset of melting, $T_{\mathrm{m}}$ - melting point, and $\phi_{\mathrm{m}}$ - mass-fraction crystallinity of the polymer. Right inset shows the chemical structure of P4MP. Left inset shows glass transition at $26^{\circ} \mathrm{C}$. Dotted colored lines indicate the temperatures of deformation. and matched those used for preparing biaxially stretched films for dielectric capacitors in our previous work [1].

The mechanical response for stretched P4MP films is shown by the true stress-true (Hencky) strain curves in Figure 2a. At 100 and $150^{\circ} \mathrm{C}$, a Hencky strain of 1.55 was imposed without rupturing the sample. At 22 and $50^{\circ} \mathrm{C}$, rupturing occurred near the clamps at Hencky strains of 0.86 and 1.24 , respectively. An elastic response at lower strains and strain-hardening at higher strains was observed at all deformation temperatures. The elastic modulus and tensile strength naturally decreased upon increasing the deformation temperature, however the decrease was higher on increasing the temperature from 22 to $50^{\circ} \mathrm{C}$ passing through $T_{\mathrm{g}}$. Interestingly, no necking was observed visually while stretching P4MP films and the signature for necking in the stress-strain curve (i.e. a yield point followed by plastic flow indicated by a plateau in stress) is also absent in Figure 2a. This is an unusual observation from a semi-crystalline polyolefin. The mechanical response is similar to amorphous polymers such as polystyrene and poly(methyl methacrylate) when stretched above their respective $T_{\mathrm{g}}[11,12]$. Clearly, the stretching behavior of P4MP is dominated by the amorphous regions below and above the $T_{\mathrm{g}}$, which is not surprising considering that the crystallinity in P4MP films is only $18 \%$.

The optical behavior of P4MP films is shown in Figure $2 b$ and Figure $2 c$ where in-plane birefringence is plotted as a function of Hencky strain and true stress, respectively. The intrinsic birefringence $\left(\Delta n^{0}=\right.$ $n_{\|}^{0}-n_{\perp}^{0}$ where $n_{\|}^{0}$ and $n_{\perp}^{0}$ are the refractive indices along and perpendicular to the chain backbone, respectively) of isotactic P4MP is $\sim 0.0075$ and positive for crystal modification I with a tetragonal crystal structure [13]. Before deformation, the P4MP films are unoriented as they exhibit zero birefringence. Upon deformation the birefringence increases with strain and remains positive indicating that the overall polymer chain orientation increases in the stretch/ machine direction (MD). While an increase in birefringence in the MD is expected, what is very surprising is that the birefringence is higher at a higher deformation temperature at iso-strain and iso-strain rate conditions. Such a result is counter-intuitive since polymer chains undergo faster relaxation at higher temperatures and are, therefore, expected to exhibit lower orientation in MD. In fact many semicrystalline and amorphous polymers such as low 
density polyethylene, high density polyethylene, polypropylene, polystyrene, poly(methyl methacrylate), thermoplastic polyimide etc. $[11,12,14-20]$ exhibit a decrease in birefringence with an increase in temperature when deformed above the glass-transition. The percent crystallinity of the stretched films varied between $21-24 \%$, but its dependence on the deformation temperature was not observed, hence the effect of crystallinity on the difference in birefringence of deformed films could be neglected. Additionally,
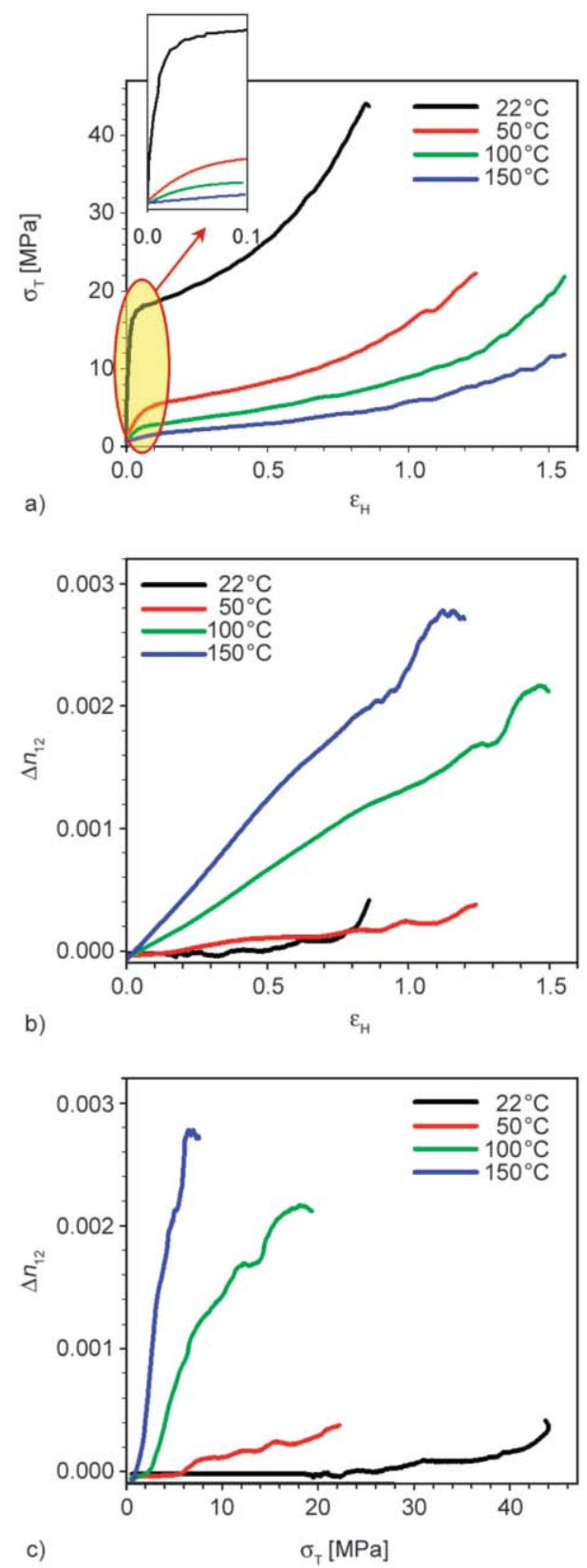

Figure 2. Deformation behavior of P4MP thin films during uniaxial stretching at $10 \mathrm{~mm} / \mathrm{min}$ at different temperatures: (a) Ttue stress-true (Hencky) strain curves, (b) strain-optic curves, and (c) stress-optic curves. it is interesting to note that the Poisson ratio for $\mathrm{P} 4 \mathrm{MP}$ is lower at lower deformation temperatures [7] i.e. the increase in free volume of the sample is higher at lower deformation temperatures. An increase in the free volume or intermolecular spacing is expected to provide a positive contribution to the overall birefringence [21], which should further increase the birefringence at lower deformation temperatures contrary to the results in Figure $2 b$ and Figure $2 \mathrm{c}$. This raises an interesting question about the physical origins of such thermally inverted strainoptical behavior in P4MP films.

The average polymer chain orientation, with birefringence as its measure, involves orientation contributions from both the crystalline and amorphous chain segments. The crystalline orientation function $\left(f_{\mathrm{c}}\right)$ at each deformation temperature was calculated by performing offline WAXD measurements on the stretched samples and using Equations (5)-(7). The wide-angle diffractogram for P4MP films before and after stretching are shown in Figure 3. P4MP film has a tetragonal crystal structure with crystal modification II [1]. Furthermore, uniform intensity rings at all Bragg diffraction angles before deformation $\left(\varepsilon_{\mathrm{H}}=0\right)$ indicates that the average crystal orientation is isotropic, which supports our result of a zero birefringence before deformation.

The wide-angle diffractograms of stretched polymer films show sharp diffraction peaks at all Bragg angles indicating that the crystalline structure in the stretched films exhibits orientation. The azimuthal spread of the diffraction peaks decreases with an increase in average polymer orientation (birefringence). It is interesting to note from Figure 3 that the crystal modification II in P4MP films before stretching transforms to a more stable crystal modification I after stretching. Also, the wide-angle diffractograms of P4MP display considerable equatorial scattering from the (212) plane [22] which increases with an increase in the polymer chain orientation. No such scattering is observed in P4MP films before deformation. This result indicates that the crystal structure becomes less perfect and disordered upon stretching even though it is thermodynamically more stable. The crystalline orientation function $\left(f_{\mathrm{c}}\right)$ after deformation follows the same temperature-dependence as birefringence, i.e. $f_{\mathrm{c}}$ is higher at higher deformation temperatures. The WAXD results suggest that it is easier to orient the crystallites in the direction of deformation at higher temperatures up to at least $150{ }^{\circ} \mathrm{C}$. 


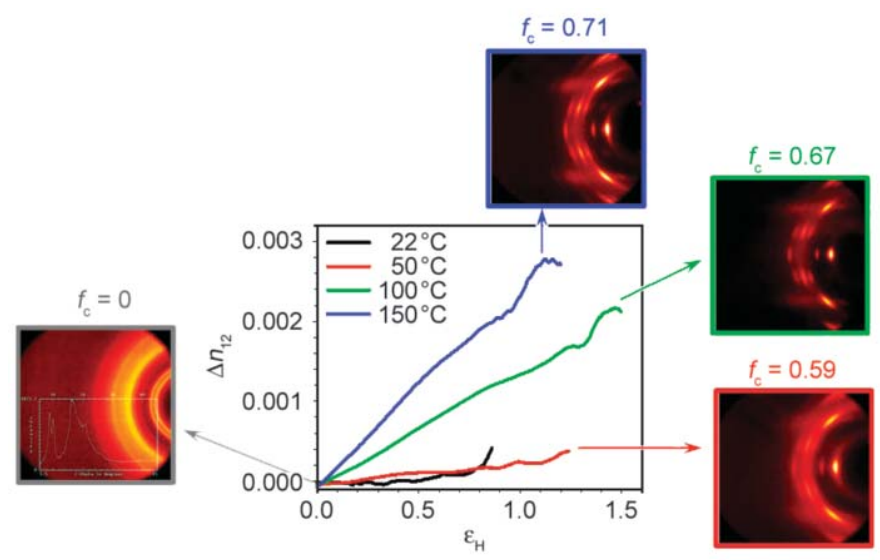

Figure 3. Wide-angle X-ray diffractograms along with the crystalline orientation function $\left(f_{\mathrm{c}}\right)$ for P4MP thin films before and after stretching at different deformation temperatures. Note the change in crystal structure of P4MP upon deformation from modification II to modification I.

In conjunction, birefringence results suggest that even the amorphous chain segments exhibit higher orientation at higher temperatures. The amorphous segments exhibit higher mobility at higher temperatures not only in the bulk phase but also on the crystal surface [23] within the crystal-amorphous transition zone. It is quite plausible that the amorphous segments orient first upon application of a force. Since these amorphous segments connect several crystallites within a network, their orientation eventually causes orientation of the crystals in the MD. Even though the amorphous segments possess higher mobility at higher temperatures, their relaxation is restricted due to confinement between several crystallites. The orientation of the crystallites then has an auto-catalytic effect of further orienting and aligning the amorphous chains in MD and so on. It is interesting to note that Richardson and Ward [24] while studying the solid-state extrusion of P4MP found that a temperature of $150^{\circ} \mathrm{C}$ was most effective in obtaining a high tensile modulus of $2.6 \mathrm{GPa}$, which is consistent with our results of obtaining the maximum chain orientation at that temperature. Infrared spectroscopy on P4MP cast and stretched P4MP films is compared in Figure 4 from 3100 $2700 \mathrm{~cm}^{-1}$. Peaks 1 and 2 at 2952 and $2922 \mathrm{~cm}^{-1}$ correspond to $\mathrm{C}-\mathrm{H}$ asymmetric stretching in $\mathrm{CH} 3$ and $\mathrm{CH}_{2}$ groups, respectively, located in the sidechain [25]. Peaks 3 and 4 at 2867 and $2848 \mathrm{~cm}^{-1}$ correspond to $\mathrm{C}-\mathrm{H}$ symmetric stretching in $\mathrm{CH}_{3}$ and $\mathrm{CH}_{2}$ side-groups, respectively. All the four peaks broadened upon stretching and their width increased upon increasing the temperature as well as the extent of deformation. This signifies some change in the environment of the side groups after stretching towards a broader distribution of vibrational energy levels. It is hard to precisely say at this point exactly what this change could mean especially because the relaxation of the side group in P4MP occurs around $-139^{\circ} \mathrm{C}$ [1]. A molecular extension of the backbone chain during uniaxial stretching of P4MP has been suggested before $[26,27]$ and it is quite possible that our observations from the FTIR measurements are a result of such a molecular extension. More precise measurements such as polarized FTIR and Raman spectroscopy will help in providing further insights

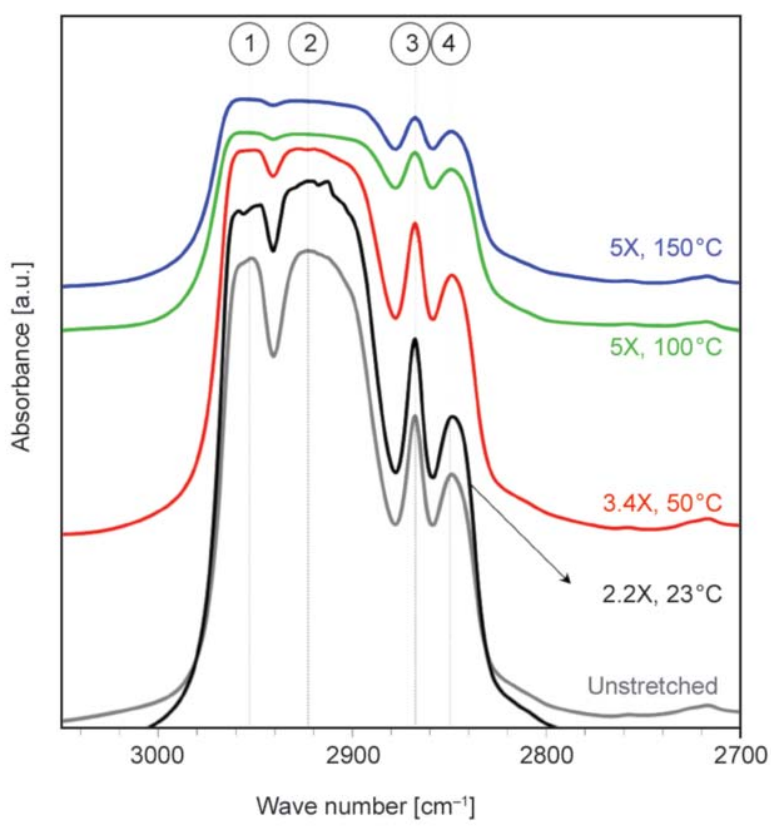

Figure 4. FTIR absorption spectra for solution-cast and uniaxially stretched P4MP thin films. Peaks 1 and 2 correspond to $\mathrm{C}-\mathrm{H}$ asymmetric stretching in $\mathrm{CH}_{3}$ and $\mathrm{CH}_{2}$ groups, respectively, located in the sidechain. Peaks 3 and 4 correspond to $\mathrm{C}-\mathrm{H}$ symmetric stretching in $\mathrm{CH}_{3}$ and $\mathrm{CH}_{2}$ groups, respectively. 
into the structural rearrangement in P4MP films in the future.

\section{Conclusions}

Real-time stress-strain-birefringence measurements reveal the thermally-inverted stress-optic and strainoptic behavior of P4MP thin films in the rubbery state. Unlike other semi-crystalline polyolefins, P4MP displays an increase in birefringence with an increase in temperature. WAXD results for the crystal orientation also show an increase in the Hermans orientation index with temperature which is consistent with birefringence measurements. These results are in contrast to the conventional knowledge that polymer chain relaxation is higher at higher temperatures and, therefore, birefringence is inversely proportional to the deformation temperatures. FTIR measurements on stretched films reveal an interesting result, where peaks corresponding to $\mathrm{C}-\mathrm{H}$ asymmetric and symmetric stretching in $\mathrm{CH}_{3}$ and $\mathrm{CH}_{2}$ side-groups display considerable broadening with respect to the unstretched film and the extent of broadening is higher for films stretched at higher temperatures. While it is hard to explain the physical origins of such thermally-inverted stress-optic and strainoptic behavior at this point, more precise measurements such as polarized FTIR and Raman spectroscopy would be helpful in providing insights in the future.

\section{Acknowledgements}

Financial support for this work through a Multidisciplinary University Research Initiative (MURI) grant from the Office of Naval Research (Contract No. N00014-10-1-0944) is greatly appreciated. The authors are thankful to Mr. Yasuhiro Higuchi at Mitsui Chemicals for providing the P4MP resin.

\section{References}

[1] Gupta S., Offenbach I., Ronzello J., Cao Y., Boggs S., Weiss R. A., Cakmak M.: Evaluation of poly(4-methyl1-pentene) as a dielectric capacitor film for high-temperature energy storage applications. Journal of Polymer Science Part B: Polymer Physics, 55, 1497-1515 (2017). https://doi.org/10.1002/polb.24399

[2] Zhang M., Zhang L., Zhu M., Wang Y., Li N., Zhang Z., Chen Q., An L., Lin Y., Nan C.: Controlled functionalization of poly(4-methyl-1-pentene) films for high energy storage applications. Journal of Materials Chemistry A, 4, 4797-4807 (2016).

https://doi.org/10.1039/C5TA09949H
[3] Ward I. M.: Optical and mechanical anisotropy in crystalline polymers. Proceedings of the Physical Society, 80, 1176-1188 (1962). https://doi.org/10.1088/0370-1328/80/5/319

[4] Janeschitz-Kreigl H.: Polymer melt rheology and flow birefringence. Springer, New York (1983).

[5] Bicakci S., Cakmak M.: Kinetics of rapid structural changes during heat setting of preoriented PEEK/PEI blend films as followed by spectral birefringence technique. Polymer, 43, 2737-2746 (2002). https://doi.org/10.1016/S0032-3861(02)00020-4

[6] Koike Y., Cakmak M.: Real time development of structure in partially molten state stretching of PP as detected by spectral birefringence technique. Polymer, 44, 42494260 (2003). https://doi.org/10.1016/S0032-3861(03)00386-0

[7] Gupta S.: Structure-property relationships in polymers for dielectric capacitors. $\mathrm{PhD}$ thesis, The University of Akron (2014).

[8] Zoller P., Starkweather H. W., Jones G. A.: The heat of fusion of poly(4-methyl pentene-1). Journal of Polymer Science Part B: Polymer Physics, 24, 1451-1458 (1986). https://doi.org/10.1002/polb.1986.090240705

[9] Alexander L. E.: X-ray diffraction methods in polymer science. Wiley, New York (1969).

[10] Brandrup J., Immergut E. H., Grulke E. A.: Polymer handbook. Wiley, New York (1999).

[11] Gupta S., Schieber J. D., Venerus D. C.: Anisotropic thermal conduction in polymer melts in uniaxial elongation flows. Journal of Rheology, 57, 427-439 (2013). https://doi.org/10.1122/1.4776237

[12] Schieber J. D., Venerus D. C., Gupta S.: Molecular origins of anisotropy in the thermal conductivity of deformed polymer melts: Stress versus orientation contributions. Soft Matter, 8, 11781-11785 (2012).

https://doi.org/10.1039/c2sm26788h

[13] Choi C-H., White J-L.: Structural changes in the melt spinning, cold drawing, and annealing of poly(4-methylpentene-1) fibers. Journal of Applied Polymer Science, 98, 130-137 (2005). https://doi.org/10.1002/app.21929

[14] Crawford S. M., Kolsky H.: Stress birefringence in polyethylene. Proceedings of the Physical Society, Section B, 64, 119-125 (1951). https://doi.org/10.1088/0370-1301/64/2/304

[15] Nakayama K., Kanetsuna H.: Hydrostatic extrusion of solid polymers. Journal of Materials Science, 10, 11051118 (1975). https://doi.org/10.1007/BF00541391

[16] Martins C. I., Cakmak M.: Large deformation mechanooptical and dynamical phase behavior in uniaxially stretched poly(ethylene naphthalate). Macromolecules, 38, 4260-4273 (2005). https://doi.org/10.1021/ma047499s 
[17] Blundell D. J., Mahendrasingam A., Martin C., Fuller W., MacKerron D. H., Harvie J. L., Oldman R. J., Riekel C.: Orientation prior to crystallisation during drawing of poly(ethylene terephthalate). Polymer, 41, 7793-7802 (2000). https://doi.org/10.1016/S0032-3861(00)00128-2

[18] Shindo Y., Hanabusa H.: Temperature dependence of birefringence in oriented poly(methyl methacrylate). Journal of Polymer Science: Polymer Letters Edition, 21, 481-486 (1983). https://doi.org/10.1002/pol.1983.130210613

[19] Offenbach I., Gupta S., Chung T. C. M., Weiss R. A., Cakmak M.: Real-time infrared-mechano-optical behavior and structural evolution of polypropylene and hydroxyl-functionalized polypropylene during uniaxial deformation. Macromolecules, 48, 6294-6305 (2015). https://doi.org/10.1021/acs.macromol.5b01017

[20] Offenbach I., Gupta S., Ma R., Treich G., Sotzing G. A., Weiss R. A., Cakmak M.: Evolution of structural mechanisms in thermoplastic polyimide (BTDA-DAH) from amorphous precursors as revealed by real-time uniaxial mechano-optical behavior. Polymer, 134, 24-34 (2018). https://doi.org/10.1016/j.polymer.2017.11.052

[21] Hong S-D., Chung S. Y., Fedors R. F., Moacanin J.: Study of molecular deformation mechanisms in the glassy state. I. Temperature effect on stress-birefringence and strain-birefringence responses of poly(methyl methacrylate). Journal of Polymer Science: Polymer Physics Edition, 21, 1647-1660 (1983).

https://doi.org/10.1002/pol.1983.180210905
[22] Charlet G., Delmas G.: Effect of solvent on the polymorphism of poly(4-methylpentene-1): 2. Crystallization in semi-dilute solutions. Polymer, 25, 1619-1625 (1984). https://doi.org/10.1016/0032-3861(84)90156-3

[23] Eng S-B., Woodward A. E.: Broad-line NMR investigation of the amorphous component of poly(trans-1,4butadiene) and poly(4-methyl pentene-1) crystals wetted by carbon disulfide. Journal of Macromolecular Science Part B: Physics, 10, 627-639 (1974). https://doi.org/10.1080/00222347408219410

[24] Richardson A., Ward I. M.: Preparation of oriented poly-4-methyl pentene-1 rods by die drawing. Polymer Communication, 28, 274-276 (1987).

[25] Samuel E. J. J., Mohan S.: FTIR and FT Raman spectra and analysis of poly(4-methyl-1-pentene). Spectrochimica Acta Part A: Molecular and Biomolecular Spectroscopy, 60, 19-24 (2004). https://doi.org/10.1016/S1386-1425(03)00212-9

[26] He T., Porter R. S.: Uniaxial draw of poly(4-methylpentene-1) by solid-state coextrusion. Polymer, 28, 946950 (1987). https://doi.org/10.1016/0032-3861(87)90167-4

[27] Osawa S., Porter R. S.: Equibiaxial deformation of poly(4-methyl-1-pentene) by a forging process. Journal of Polymer Science Part B: Polymer Physics, 32, 535540 (1994). https://doi.org/10.1002/polb.1994.090320314 